\title{
The influence of user characteristics on spatial perception differences in 3D visual environments
}

\author{
Lina Huang $^{\mathrm{a},}$ *, Yanfang Liu ${ }^{\mathrm{a}}$, Shen Ying ${ }^{\mathrm{a}}$ \\ ${ }^{a}$ School of Resource and Environmental Science, Wuhan University, Lina Huang(linahuang@whu.edu.cn), Yanfang \\ Liu(yfliu610@163.com), Shen Ying(shy@whu.edu.cn) \\ * Corresponding author
}

Keywords: spatial perception, user difference, 3D visual environment, pedestrian navigation

\begin{abstract}
:
With the rapid development of 3D technology currently, in particular, on the context of building smart cities, a number of novel 3D geo-visualizations have been advanced, such as immersive maps, panoramic location-based service, and augmented reality systems. These visualizations refer to not only the technological aspects but also the user's abilities to cognize and make used of presented information(Herman et al., 2018, Šašinka et al., 2018, Kubíček et al., 2019). Note the existing researches usually concentrate on the presentation parameters and the usability of consequential visualizations in actual tasks(Lokka and Coltekin, 2016, Roth et al., 2017, Lokka and Coltekin, 2019). The influence of user factors on 3D spatial perception which is essential for 3D geo-visualization applications still remains unclear.
\end{abstract}

In our study, we explored the user differences of spatial perception in 3D geo-visualization in contrast to that in real scene. The research questions were specified as follows: (1) How does the user factors, i.e. gender, age, academic background, mental rotation ability, and abstract reasoning ability, influence the user performance in spatial tasks related to distance perception, height perception, and environmental perception(Siegel and White, 1975)? (2) Is the user perception ability in $3 \mathrm{D}$ geo-visualization consistent with that in real scene?

This study is an exploratory research. Series of experiments were designed based on questionnaire survey, mental mapping and eye-tracking techniques. On the one hand, a questionnaire, a Mental Rotation Test (MRT) and an Abstract Reasoning Test (ART) were conducted to figure out the user profiles, namely the personal information of the participants. On the other hand, a list of spatial tasks were instructed both in a 3D geo-visualization and in a real scene. During the procedure, participants were requested to answer several questions, in addition, their eye movements were recorded using an eye tracker. The participants were also asked to draw a mental map with hands to recall the layout of scene and their travelling path. After all these experiments, the participants' spatial perception ability were assessed by calculating the accuracy and efficiency of their performance. Consequentially, the significance of participant differences were investigated through a set of quantitative analysis.

The experimental process refers to three stages: Firstly the questionnaire including MRT and ART, secondly spatial performance in a virtual 3D scene, and thirdly comparative spatial performance in the real scene. For the stage of virtual 3D scene, an interactive 3D geo-visualization platform was developed, so that the users can freely switch the Line of Sight (LOS) and Angle of Field (AOF). For the stage of real scene, a real-world area which is isomorphic to the virtual 3D scene was designated as the test filed. Forty participants were recruited from three universities in Wuhan, China. According to our study, a few interesting results can be obtained:

(1) In general, there are significant user differences in spatial perception with respect to the visual style, i.e. 3D geovisualization and real scene. The participants expressed higher level of environmental perception in real scene than in 3D geo-visualization $\left(\mathrm{r}_{-3 \mathrm{~d}}=4.333, \mathrm{r}_{\text {real }}=4.001\right.$, Sig. $\left.=0.044\right)$. The participants provided mental maps of real scene in more details than that of the 3D geo-visualization. Meanwhile, they perform better way-finding behaviour with shorter distance moving and less corner turning in real scene.

(2) By measuring the single influence of user characters, it is indicated that orientation idiom has significant effect on user's mental rotation ability. The participants who use front-back-left-right as their orientation idioms trend to have higher correct accuracy in MRT, while those using north-south-east-west as their orientation idiom seem to have lower correct accuracy ( $\mathrm{r}_{- \text {fbll}}=19.64, \mathrm{r}_{\text {_nsew }}=12.46$, Sig. $=0.030$, Independent-samples Mann-Whitney U Test).

When we group participants with the spatial reference frameworks of self-centred reference, fixed reference and coordinative reference(Byrne et al., 2007), it is easy to find that the type of spatial reference frameworks has relative significant effect on distance perception in 3D geo-visualization. Those participants using self-centred reference show the highest accuracy rate, while the ones using coordinative reference show relative lowest accuracy rate $\left(\mathrm{r}_{\text {_selfCent }}=22.42, \mathrm{r}_{\text {_fixed }}=15.47, \mathrm{r}_{\text {_coord }}=13.43\right.$, Sig. $=0.056$, Independent-samples Kruskal-Wallis Test $)$. 
Nevertheless, other user factors, including academic background (students of cartography or non-cartography), age (from 21-24) and activity scope, show no significant influence on spatial perception in 3D geo-visualization as well as in real scene.

(3) Looking into the combined influence of multiple factors, there is a significant interaction between gender and orientation idioms on the environmental perception in 3D geo-visualization environment, but neither of them shows a significant main effect (Sig._gender $r=0.817$, Sig._orildio $=0.423$, Sig._combined $=0.037$ ). The female participants using north-south-east-west orientation idiom have higher level of environmental perception than those using frontback-left-right (Sig.=0.064), however, the male participants show no significant difference no matter which orientation idiom they use.

Spatial reference framework and orientation idiom have similar significant interaction on distance perception in real scene, furthermore, the orientation idiom presents a relative significant main effect (Sig._spatialRef $=0.882$, Sig._orildio $=0.071$, Sig._combined $=0.038$ ). The participants who use self-centred reference and orientation idiom of north-south-east-west have higher accuracy rate than the ones using self-centred reference and orientation idiom of front-back-left-right (Sig.=0.007).

In addition, gender and spatial reference framework seem have relative significant interaction on distance perception in 3D geo-visualization, and the spatial reference framework provides a main effect significantly (Sig._gende $\mathrm{r}=0.223$, Sig._spatialRef $=0.019$, Sig._combined $=0.077$ ).

These experimental results provide a bright prospect to improve the $3 \mathrm{D}$ geo-visualizations to fit users' personalized charactoristics for certain spatial tasks. They will also be beneficial to the design of mixed 3D geo-visualization, e.g. immersive maps and augmented reality systems, that combines the advantages of visual 3D scene and real scene.

\section{References:}

Byrne, P., Becker, S. and Burgess, N., 2007. Remembering the past and imagining the future: A neural model of spatial memory and imagery. Psychological review, 114 (2), 340-375. doi:10.1037/0033-295x.114.2.340

Herman, L., et al., 2018. Evaluation of user performance in interactive and static $3 \mathrm{~d}$ maps. ISPRS International Journal of Geo-Information, 7, 415. doi:10.3390/ijgi7110415

Kubíček, P., et al., 2019. Identification of altitude profiles in 3d geovisualizations: The role of interaction and spatial abilities. International Journal of Digital Earth, 12 (2), 156-172. doi:10.1080/17538947.2017.1382581

Lokka, I. and Coltekin, A., Year. Simulating navigation with virtual 3d geovisualizations - a focus on memory related factorsed.^eds. XXIII ISPRS Congress, Commission II, Prague, 671-673.

Lokka, I. and Coltekin, A., 2019. Toward optimizing the design of virtual environments for route learning: Empirically assessing the effects of changing levels of realism on memory. International Journal of Digital Earth, 12 (2), $137-155$. doi:10.1080/17538947.2017.1349842

Roth, R., et al., 2017. User studies in cartography: Opportunities for empirical research on interactive maps and visualizations. International Journal of Cartography, 3 (sup1), 61-89. doi:10.1080/23729333.2017.1288534

Šašinka, Č., et al., 2018. Collaborative immersive virtual environments for education in geography. ISPRS International Journal of Geo-Information, 8 (1), 3. doi:10.3390/ijgi8010003

Siegel, A.W. and White, S.H., 1975. The development of spatial representations of large-scale environments. Advances in Child Development \& Behavior, 10, 9-55 